\title{
Sharp Smith's bounds for the gamma function
}

\author{
Xi-Qiao Li' , Zhi-Ming Liu' ${ }^{1}$, Zhen-Hang Yang ${ }^{2}$ and Shen-Zhou Zheng ${ }^{3 *}$ (D)
}

*Correspondence:

shzhzheng@bjtu.edu.cn

${ }^{3}$ College of Science, Beijing

Jiaotong University, Beijing, China

Full list of author information is

available at the end of the article

\section{Abstract}

Among various approximation formulas for the gamma function, Smith showed that

$$
\Gamma\left(x+\frac{1}{2}\right) \sim S(x)=\sqrt{2 \pi}\left(\frac{x}{e}\right)^{x}\left(2 x \tanh \frac{1}{2 x}\right)^{x / 2}, x \rightarrow \infty
$$

which is a little-known but accurate and simple one. In this note, we prove that the function $x \mapsto \ln \Gamma(x+1 / 2)-\ln S(x)$ is strictly increasing and concave on $(0, \infty)$, which shows that Smith's approximation is just an upper one.

MSC: Primary 33B15; 26A48; secondary 26D15; 26A51

Keywords: Gamma function; Approximation formula; Inequality

\section{Introduction}

The Stirling formula

$$
n ! \sim \sqrt{2 \pi n} n^{n} e^{-n}
$$

has many important applications in statistical physics, probability theory and number theory. Due to its practical importance, it has attracted much interest of many mathematicians and has also motivated a large number of research papers concerning various generalizations and improvements; see for example, Burnside's [1], Gosper [2], Batir [3], Mortici [4].

The gamma function $\Gamma(x)=\int_{0}^{\infty} t^{x-1} e^{-t} d t$ for $x>0$ is closely related to the Stirling formula, since $\Gamma(n+1)=n$ ! for all $n \in \mathbb{N}$. This inspired some authors to also pay attention to find various better approximations for the gamma function; see, for instance, Ramanujan [5, p. 339], Windschitl (see Nemes [6, Corollary 4.1]), Yang and Chu [7], Chen [8].

More results involving the approximation formulas for the factorial or gamma function can be found in [9-23] and the references cited therein.

In this note, we are interested in Smith's approximation formula (see [24, equation (42)]):

$$
\Gamma\left(x+\frac{1}{2}\right) \sim \sqrt{2 \pi}\left(\frac{x}{e}\right)^{x}\left(2 x \tanh \frac{1}{2 x}\right)^{x / 2}:=S(x), \quad \text { as } x \rightarrow \infty .
$$

(c) The Author(s) 2018. This article is distributed under the terms of the Creative Commons Attribution 4.0 International License (http://creativecommons.org/licenses/by/4.0/), which permits unrestricted use, distribution, and reproduction in any medium, provided you give appropriate credit to the original author(s) and the source, provide a link to the Creative Commons license, and indicate if changes were made. 
It is easy to check that

$$
\Gamma\left(x+\frac{1}{2}\right)=\sqrt{2 \pi}\left(\frac{x}{e}\right)^{x}\left(2 x \tanh \frac{1}{2 x}\right)^{x / 2}\left(1+O\left(\frac{1}{x^{5}}\right)\right),
$$

which shows that the rate of $S(x)$ converging to $\Gamma(x+1 / 2)$ as $x \rightarrow \infty$ is like $x^{-5}$. According to the comment in $[8,(3.5)-(3.10)]$, it is well known that Smith's approximation is an accurate but simple one for gamma function.

The aim of this short note is to further prove the Smith approximation $S(x)$ is an upper one. Our main result is stated as follows.

Theorem 1 The function

$$
f(x)=\ln \Gamma\left(x+\frac{1}{2}\right)-\ln \sqrt{2 \pi}-x \ln x+x-\frac{x}{2} \ln \left(2 x \tanh \frac{1}{2 x}\right)
$$

is strictly increasing and concave from $(0, \infty)$ onto $(-\ln \sqrt{2}, 0)$.

\section{Proof of Theorem 1}

To prove Theorem 1 we need the following two lemmas.

Lemma 1 The inequality

$$
\psi^{\prime}\left(x+\frac{1}{2}\right)<\frac{4}{3} \frac{15 x^{2}+4}{x\left(20 x^{2}+7\right)}
$$

holds for all $x>0$.

Proof Let

$$
f_{1}(x)=\psi^{\prime}\left(x+\frac{1}{2}\right)-\frac{4}{3} \frac{15 x^{2}+4}{x\left(20 x^{2}+7\right)}
$$

Using the recurrence formula [25, pp. 258-260]:

$$
\psi^{(n)}(x+1)-\psi^{(n)}(x)=\frac{(-1)^{n} n !}{x^{n+1}}
$$

we have

$$
\begin{aligned}
f_{1}(x+1)-f_{1}(x)= & \psi^{\prime}\left(x+\frac{3}{2}\right)-\frac{4}{3(x+1)} \frac{15 x^{2}+30 x+19}{20 x^{2}+40 x+27} \\
& -\psi^{\prime}\left(x+\frac{1}{2}\right)+\frac{4}{3} \frac{15 x^{2}+4}{x\left(20 x^{2}+7\right)} \\
& -\frac{1}{(x+1 / 2)^{2}}-\frac{4}{3(x+1)} \frac{15 x^{2}+30 x+19}{20 x^{2}+40 x+27}+\frac{4}{3} \frac{15 x^{2}+4}{x\left(20 x^{2}+7\right)} \\
= & \frac{144}{x(x+1)(2 x+1)^{2}\left(20 x^{2}+7\right)\left(20 x^{2}+40 x+27\right)}>0 .
\end{aligned}
$$


It then follows that

$$
f_{1}(x)<f_{1}(x+1)<\cdots<\lim _{n \rightarrow \infty} f_{1}(x+n)=0
$$

which proves the desired inequality (2.1).

Lemma 2 The inequality

$$
\frac{\sinh ^{2} t}{\cosh t}>\frac{t^{2}\left(21 t^{2}+60\right)}{31 t^{2}+60}
$$

holds for all $t>0$.

Proof It is obvious that the inequality what we consider is equivalent to

$$
f_{2}(t)=\left(31 t^{2}+60\right)(\sinh t)^{2}-t^{2}\left(21 t^{2}+60\right) \cosh t>0 .
$$

Simplifying and expanding it in power series lead us to

$$
\begin{aligned}
2 f_{2}(t)= & 60 \cosh 2 t+31 t^{2} \cosh 2 t-120 t^{2} \cosh t-42 t^{4} \cosh t-31 t^{2}-60 \\
= & 60 \sum_{n=0}^{\infty} \frac{2^{2 n}}{(2 n) !} t^{2 n}+31 \sum_{n=1}^{\infty} \frac{2^{2 n-2}}{(2 n-2) !} t^{2 n} \\
& -120 \sum_{n=1}^{\infty} \frac{1}{(2 n-2) !} t^{2 n}-42 \sum_{n=2}^{\infty} \frac{1}{(2 n-4) !} t^{2 n}-31 t^{2}-60 \\
:= & \sum_{n=2}^{\infty} \frac{a_{n}}{(2 n) !} t^{2 n}
\end{aligned}
$$

where

$$
a_{n}=\left(62 n^{2}-31 n+120\right) 2^{2 n-1}-24 n(2 n-1)\left(14 n^{2}-35 n+31\right) .
$$

It is easy to check that $a_{2}=a_{3}=0$ and $a_{4}=49184>0$. It remains to prove $a_{n}>0$ for $n \geq 5$.

To this end, it suffices to prove $b_{n}=2^{2 n-1}-6 n(2 n-1)>0$ for $n \geq 5$, because the inequality

$$
\left(62 n^{2}-31 n+120\right)>4\left(14 n^{2}-35 n+31\right)
$$

is clearly valid for $n \geq 5$. We easily obtain

$$
b_{n+1}-4 b_{n}=6\left(6 n^{2}-7 n-1\right)>0
$$

for $n \geq 5$, which in combination with $b_{5}=242>0$ yields $b_{n}>0$ for $n \geq 5$. This completes the proof.

Now we are in a position to prove Theorem 1. 
Theorem 1 Differentiating and simplifying yields

$$
\begin{aligned}
& f^{\prime}(x)=\psi\left(x+\frac{1}{2}\right)-\ln x-\frac{1}{2} \ln \left(2 x \tanh \frac{1}{2 x}\right)+\frac{1}{2 x \sinh (1 / x)}-\frac{1}{2} \\
& f^{\prime \prime}(x)=\psi^{\prime}\left(x+\frac{1}{2}\right)+\frac{1}{2 x^{3}} \frac{\cosh (1 / x)}{\sinh ^{2}(1 / x)}-\frac{3}{2 x}
\end{aligned}
$$

As an application of inequalities (2.1) and (2.3) it gives

$$
\begin{aligned}
f^{\prime \prime}(x) & <\frac{4}{3} \frac{15 x^{2}+4}{x\left(20 x^{2}+7\right)}+\frac{1}{2 x^{3}} \frac{\cosh (1 / x)}{\sinh ^{2}(1 / x)}-\frac{3}{2 x} \\
& =\frac{1}{2 x^{3}} \frac{\cosh (1 / x)}{\sinh ^{2}(1 / x)}-\frac{1}{6} \frac{60 x^{2}+31}{x\left(20 x^{2}+7\right)} \\
& \stackrel{x}{x=1 / t} \frac{t^{3}}{2}\left(\frac{\cosh t}{\sinh ^{2} t}-\frac{31 t^{2}+60}{t^{2}\left(21 t^{2}+60\right)}\right)<0 .
\end{aligned}
$$

Then it is deduced that

$$
f^{\prime}(x)>\lim _{x \rightarrow \infty} f^{\prime}(x)=0
$$

which in turn implies that

$$
-\frac{1}{2} \ln 2=\lim _{x \rightarrow 0^{+}} f(x)<f(x)<\lim _{x \rightarrow \infty} f(x)=0 .
$$

This completes the proof.

\section{Corollaries and remarks}

Using the increasing property of $f(x+1 / 2)$ given in Theorem 1 and noting that

$$
f\left(\frac{1}{2}\right)=\ln \frac{\sqrt{e}}{\sqrt{\pi}(\tanh 1)^{1 / 4}} \quad \text { and } \quad f\left(\frac{3}{2}\right)=\ln \left(\frac{2 e \sqrt{e} 3^{3 / 4}}{27 \sqrt{\pi} \tanh ^{3 / 4}(1 / 3)}\right)
$$

we have the corollaries.

Corollary 1 The double inequality

$$
\alpha_{1}<\frac{e^{x+1 / 2} \Gamma(x+1)}{\sqrt{2 \pi}(x+1 / 2)^{x+1 / 2}[(2 x+1) \tanh (1 /(2 x+1))]^{(2 x+1) / 4}}<1
$$

holds for all $x>0$ with the best constants 1 and $\alpha_{1}=\sqrt{e / \pi} /(\tanh 1)^{1 / 4} \approx 0.99573$.

Corollary 2 The double inequality

$$
\alpha_{2}<\frac{n !}{\sqrt{2 \pi}((n+1 / 2) / e)^{n+1 / 2}[(2 n+1) \tanh (1 /(2 n+1))]^{(2 n+1) / 4}}<1
$$

holds for all $n \in \mathbb{N}$ with the best constants 1 and

$$
\alpha_{2}=\frac{2 e \sqrt{e} 3^{3 / 4}}{27 \sqrt{\pi} \tanh ^{3 / 4}(1 / 3)} \approx 0.99994
$$


By the decreasing property of $f^{\prime}(x+1 / 2)$ given in Theorem 1 and the facts that

$$
\begin{aligned}
& f^{\prime}\left(\frac{1}{2}\right)=\frac{1}{\sinh 2}-\frac{1}{2} \ln (\tanh 1)+\ln 2-\frac{1}{2}-\gamma \approx 0.027823 \\
& f^{\prime}\left(\frac{3}{2}\right)=\frac{1}{3 \sinh (2 / 3)}-\frac{1}{2} \ln \left(3 \tanh \frac{1}{3}\right)-\ln \frac{3}{2}+\psi(1)+\frac{1}{2} \approx 0.00016946
\end{aligned}
$$

the following corollaries are immediate.

Corollary 3 For $x>0$, the inequalities

$$
\begin{aligned}
\frac{1}{2} & +\frac{1}{2} \ln \left((2 x+1) \tanh \frac{1}{2 x+1}\right)-\frac{1}{(2 x+1) \sinh (2 /(2 x+1))} \\
& <\psi(x+1)-\ln \left(x+\frac{1}{2}\right) \\
& <\beta_{1}+\frac{1}{2} \ln \left((2 x+1) \tanh \frac{1}{2 x+1}\right)-\frac{1}{(2 x+1) \sinh (2 /(2 x+1))}
\end{aligned}
$$

hold, where the constants $1 / 2$ and

$$
\beta_{1}=\ln 2-\frac{1}{2} \ln (\tanh 1)+\frac{1}{\sinh 2}-\gamma \approx 0.52782
$$

are the best possible.

Corollary 4 Let $H_{n}=\sum_{k=1}^{n}$ for $n \in \mathbb{N}$. The inequalities

$$
\begin{aligned}
& \left(\frac{1}{2}+\gamma\right)+\frac{1}{2} \ln \left((2 n+1) \tanh \frac{1}{2 n+1}\right)-\frac{1}{(2 n+1) \sinh (2 /(2 x+1))} \\
& <H_{n}-\ln \left(n+\frac{1}{2}\right) \\
& <\beta_{2}+\frac{1}{2} \ln \left((2 n+1) \tanh \frac{1}{2 n+1}\right)-\frac{1}{(2 n+1) \sinh (2 /(2 n+1))}
\end{aligned}
$$

hold, where $1 / 2+\gamma \approx 1.0772$ and

$$
\beta_{2}=\frac{1}{3 \sinh (2 / 3)}-\frac{1}{2} \ln \left(3 \tanh \frac{1}{3}\right)-\ln \frac{3}{2}+1 \approx 1.0774
$$

are the best possible constants.

Finally, as a by-product of Lemma 1, we draw the following conclusion.

Theorem 2 Let $g$ be defined on $(0, \infty)$ by

$$
g(x)=\ln \Gamma\left(x+\frac{1}{2}\right)-\left[\frac{1}{2} \ln 2 \pi+\frac{16}{21} x \ln x+\frac{5 x}{42} \ln \left(x^{2}+\frac{7}{20}\right)-x-\frac{\sqrt{35}}{42} \operatorname{arccot}\left(\sqrt{\frac{20}{7}} x\right)\right]
$$

Then $g$ is strictly increasing and concave on $(0, \infty)$. 
Proof Differentiation yields

$$
\begin{aligned}
& g^{\prime}(x)=\psi\left(x+\frac{1}{2}\right)-\left[\frac{5}{42} \ln \left(x^{2}+\frac{7}{20}\right)+\frac{16}{21} \ln x\right], \\
& g^{\prime \prime}(x)=\psi^{\prime}\left(x+\frac{1}{2}\right)-\frac{4}{3} \frac{15 x^{2}+4}{x\left(20 x^{2}+7\right)}=f_{1}(x)<0,
\end{aligned}
$$

where the inequality holds due to Lemma 1 . This completes the proof.

Remark 1 Theorem 2 gives a new approximation for the gamma function

$$
\Gamma\left(x+\frac{1}{2}\right) \sim \sqrt{2 \pi} x^{26 x / 21}\left(x^{2}+\frac{7}{20}\right)^{5 x / 42} \exp \left[-x-\frac{\sqrt{35}}{42} \operatorname{arccot}\left(\sqrt{\frac{20}{7}} x\right)\right] \text {, }
$$

as $x \rightarrow \infty$, which satisfies

$$
\Gamma\left(x+\frac{1}{2}\right)=\sqrt{2 \pi} x^{26 x / 21}\left(x^{2}+\frac{7}{20}\right)^{5 x / 42} \exp \left[-x-\frac{\sqrt{35}}{42} \operatorname{arccot}\left(\sqrt{\frac{20}{7}} x\right)\right]\left(1+O\left(x^{-5}\right)\right) .
$$

Remark 2 Theorem 2 also offers an asymptotic formula for the psi function

$$
\psi\left(x+\frac{1}{2}\right) \sim \frac{5}{42} \ln \left(x^{2}+\frac{7}{20}\right)+\frac{16}{21} \ln x \quad \text { as } x \rightarrow \infty
$$

Furthermore, by replacing $x$ with $x+1 / 2$, we have the following sharp inequalities:

$$
\begin{aligned}
& \frac{5}{42} \ln \left(x^{2}+x+\frac{3}{5}\right)+\frac{16}{21} \ln \left(x+\frac{1}{2}\right) \\
& \quad<\psi(x+1)<\lambda_{0}+\frac{5}{42} \ln \left(x^{2}+x+\frac{3}{5}\right)+\frac{16}{21} \ln \left(x+\frac{1}{2}\right)
\end{aligned}
$$

for $x>0$ with the best constant

$$
\begin{aligned}
\lambda_{0} & =\frac{16}{21} \ln 2-\frac{5}{42} \ln \frac{3}{5}-\gamma \approx 0.011709 \\
\gamma & +\frac{5}{42} \ln \left(n^{2}+n+\frac{3}{5}\right)+\frac{16}{21} \ln \left(n+\frac{1}{2}\right) \\
& <H_{n}<\lambda_{0}+\gamma+\frac{5}{42} \ln \left(n^{2}+n+\frac{3}{5}\right)+\frac{16}{21} \ln \left(n+\frac{1}{2}\right)
\end{aligned}
$$

for $n \in \mathbb{N}$ with the best constant

$$
\lambda_{1}=1-\frac{16}{21} \ln \frac{3}{2}-\frac{5}{42} \ln \frac{13}{5}-\gamma \approx 0.00010718
$$

Inequalities (3.1) first appeared in [26, Corollary 3.4].

\section{Conclusions}

In this note, we mainly presented an upper bound of Smith's approximation in accordance with the fact that the function $x \mapsto \ln \Gamma(x+1 / 2)-\ln S(x)$ is strictly increasing and concave on $(0, \infty)$. As a consequence, we get some new sharp estimates to various classical inequalities concerning the gamma function and hyperbolic functions. 
Acknowledgements

This paper is supported by the National Science Foundation of China grant No. 11371050.

\section{Competing interests}

The authors declare that they have no competing interests.

\section{Authors' contributions}

All authors contributed to each part of this work equally, and they all read and approved the final manuscript.

\section{Author details}

${ }^{1}$ School of Mechanical, Electronic and Control Engineering, Beijing Jiaotong University, Beijing, China. ${ }^{2}$ Department of Science and Technology, State Grid Zhejiang Electric Power Company Research Institute, Hangzhou, China. ${ }^{3}$ College of Science, Beijing Jiaotong University, Beijing, China.

\section{Publisher's Note}

Springer Nature remains neutral with regard to jurisdictional claims in published maps and institutional affiliations.

Received: 2 October 2017 Accepted: 15 January 2018 Published online: 31 January 2018

\section{References}

1. Burnside, W.: A rapidly convergent series for log N!. Messenger Math. 46, 157-159 (1917)

2. Gosper, R.W.: Decision procedure for indefinite hypergeometric summation. Proc. Natl. Acad. Sci. USA 75, 40-42 (1978)

3. Batir, N.: Sharp inequalities for factorial $n$. Proyecciones 27(1), 97-102 (2008)

4. Mortici, C:: On the generalized Stirling formula. Creative Math. Inform. 19(1), 53-56 (2010)

5. Ramanujan, S.: The Lost Notebook and Other Unpublished Papers. Springer, Berlin (1988)

6. Nemes, G.: New asymptotic expansion for the gamma function. Arch. Math. (Basel) 95, 161-169 (2010)

7. Yang, Z.H., Chu, Y.M.: Asymptotic formulas for gamma function with applications. Appl. Math. Comput. 270, 665-680 (2015)

8. Chen, C.P.: A more accurate approximation for the gamma function. J. Number Theory 164, 417-428 (2016)

9. Shi, X., Liu, F., Hu, M.: A new asymptotic series for the gamma function. J. Comput. Appl. Math. 195, 134-154 (2006)

10. Guo, B.N., Zhang, Y.J., Qi, F.: Refinements and sharpenings of some double inequalities for bounding the gamma function. J. Inequal. Pure Appl. Math. 9(1), Article ID 17 (2008)

11. Batir, N.: Inequalities for the gamma function. Arch. Math. 91, 554-563 (2008)

12. Mortici, C.: An ultimate extremely accurate formula for approximation of the factorial function. Arch. Math. 93(1), $37-45(2009)$

13. Mortici, C.: New sharp inequalities for approximating the factorial function and the digamma functions. Miskolc Math. Notes 11(1), 79-86 (2010)

14. Mortici, C.: Improved asymptotic formulas for the gamma function. Comput. Math. Appl. 61, 3364-3369 (2011)

15. Zhao, J.L., Guo, B.N., Qi, F.: A refinement of a double inequality for the gamma function. Publ. Math. (Debr.) 80(3-4), 333-342 (2012)

16. Mortici, C:: Further improvements of some double inequalities for bounding the gamma function. Math. Comput. Model. 57, 1360-1363 (2013)

17. Feng, L., Wang, W.: Two families of approximations for the gamma function. Numer. Algorithms 64, 403-416 (2013)

18. Qi, F: Integral representations and complete monotonicity related to the remainder of Burnside's formula for the gamma function. J. Comput. Appl. Math. 268, 155-167 (2014)

19. Lu, D.: A new sharp approximation for the gamma function related to Burnside's formula. Ramanujan J. 35(1), 121-129 (2014)

20. Yang, Z.H., Tian, J.F.: Monotonicity and inequalities for the gamma function. J. Inequal. Appl. 2017, Article ID 317 (2017)

21. Yang, Z.H., Tian, J.F.: Monotonicity and sharp inequalities related to gamma function. J. Math. Inequal. 12(1) (2018). https://doi.org/10.7153/jmi-2018-12-01

22. Alzer, H.: On some inequalities for the gamma and psi functions. Math. Comput. 66(217), 373-389 (1997)

23. Yang, Z.H.: Approximations for certain hyperbolic functions by partial sums of their Taylor series and completely monotonic functions related to gamma function. J. Math. Anal. Appl. 441, 549-564 (2016)

24. Smith, W.D.: The gamma function revisited. http://schule.bayernport.com/gamma/gamma05.pdf (2006)

25. Abramowttz, M., Stegun, I.A.: Handbook of Mathematical Functions with Formulas, Graphs and Mathematical Tables. Dover, New York (1972)

26. Zhao, T.H., Yang, Z.H., Chu, Y.M.: Monotonicity properties of a function involving the psi function with applications. J. Inequal. Appl. 2015, Article ID 193 (2015) 